\title{
Within-generational and diversity-dependent effects in an individual-based model of predator-prey interaction
}

\author{
W. J. Chivers, R. D. Herbert and W. Gladstone
}

\begin{abstract}
In this paper we report the use of an individual-based model of predator-prey interaction to explore the effects of "within generational" and "between generational" updating of a system level variable. We also report the importance of diversity within the simulated populations. Our findings support those of Grimm and Uchmański [1994] in regard to the importance of the timing of system level variables, and support Grimm and Uchmański and others in regard to the importance of the level of diversity across the population. The significance of these findings is emphasized by the fundamental differences between our model and that of Grimm and Uchmański in regard to the assumptions made about resource flow in the system.
\end{abstract}

\section{Introduction}

The use of individual-based modeling (IBM) in ecology is an alternative approach to the more traditional mathematical or state variable approach in which ordinary or partial differential equations are used to simulate system outcomes over time. An IBM consists of a set of heterogeneous, discrete objects which interact with each other and their environment. Execution of the model involves simulating local interactions between individuals and the environment. In contrast, traditional mathematical modeling takes a top-down approach, expressing relationships between global outcomes and modeling populations, rather than individuals.

A significant difference between individual-based models and mathematical models is in the mechanism by which system-level behavior emerges. In an individual-based model, system level outcomes such as population levels or carrying capacity emerge from the local, discrete interactions involving the heterogeneous and discrete individuals. These interactions and their effects can be implemented in real time in an individual-based model, with immediate effects on system variables such as population level. The model described in this paper, for example, can update the herbivore population variable as each herbivore is 
eaten, which may occur many times in one cycle or time step. In contrast, a characteristic of normal population-level mathematical models is their implementation in discrete time steps and the consequent updating of system level variables at those discrete times.

The issue of the timing of system level variable updating has received little attention in the literature. One exception is the work of Grimm and Uchmański [1994] who find it an important influence in the dynamics of an individual-based model of a single population which employs a simple resource flow mechanism in which unspecified resources flow into their system at a constant rate and are distributed at a predictable rate between the individuals. The artificial model of resource dynamics and the use of only one population by Grimm and Uchmański leaves open the question of whether their conclusions apply to other, more complex models.

The model described in this paper employs somewhat more realistic resource inflow and distribution between two interacting species. Despite the differences in our models, our findings support those of Grimm and Uchmański that the difference between "within-generational" and "between-generational" variable updating is very significant for the dynamics of the model. Our findings also support their suggestion that variability across populations is important for the model dynamics. The importance of the latter in this model was reported in Chivers and Herbert [2003] and is mentioned again here in the context of the comparison of these models.

\section{The individual-based model}

The individual-based model of predator-prey interaction used here is described in detail in Chivers and Herbert [2003], and the following is an abbreviated description. Each predator and prey individual is discretely represented in the computer memory, and each individual maintains a resource level which is a simple analogy of the energy reserves stored by living organisms. Resources flow from the environment to the prey and then to the predators as the prey are eaten. The concept of using resources as the currency of the model was inspired by the Gecko model of Schmitz and Booth [Schmitz and Booth, 1997]. The two species occupy an homogeneous environment space and are assumed to be randomly distributed. The primary output of the model is the population levels of the two species over time.

Processing of each individual occurs each cycle or time step. This involves resource intake - the predators eat prey to acquire their resources, and the prey are given a random number of resources to simulate grazing or photosynthesis. A tax is then deducted from the resource total of each individual to simulate the metabolic cost of living for each cycle. Individuals die if their resource level is not sufficient. Finally individuals may produce one clonal offspring each cycle if their resource level is high enough.

The model was originally built with the goals of parsimony, replication of the Lotka-Volterra model, generalization and the use of energy resource flow as 


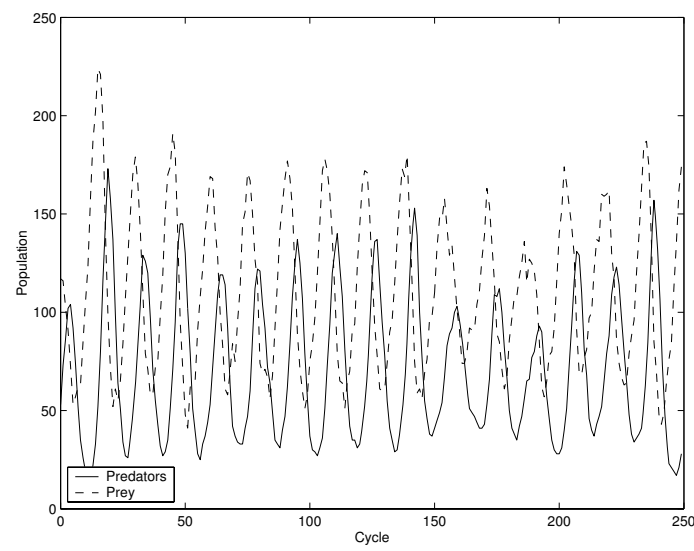

Figure 1: The individual-based model -250 cycles.

the currency of the model.

\subsection{Model output}

The interaction of the individuals generates comparable results to the LotkaVolterra model, albeit with characteristics of stochastic and chaotic systems. Consistent population maxima and minima, while not explicitly coded into the IBM, emerge from the heterogeneous individuals and their rules of engagement, and these population levels are resistant to stochastic population fluctuations and exogenous shock.

Figure 1 illustrates typical output over 250 cycles, Figure 2 over 30000 cycles.

The tendency of the model to maintain approximate population maxima and minima is generally observed across the parameter space explored, and is an illustration of the emergence of system level behavior from local interactions in an individual-based model.

\section{Within- and between-generational effects}

The model illustrated in Figures 1 and 2 implements real-time or within-generational updating of system variables - in this case the two populations. During the processing of the predator list which occurs each cycle, prey are removed from the environment as they are eaten. As the algorithm proceeds through the predator list during the processing of each cycle, then, if predator $P_{n}$ eats, the probability of predator $P_{n+1}$ eating is slightly diminished because there is one less prey individual in the environment and the probability of each predator eating is proportional to the number of prey and is calculated separately for each predator during the processing of each cycle. 


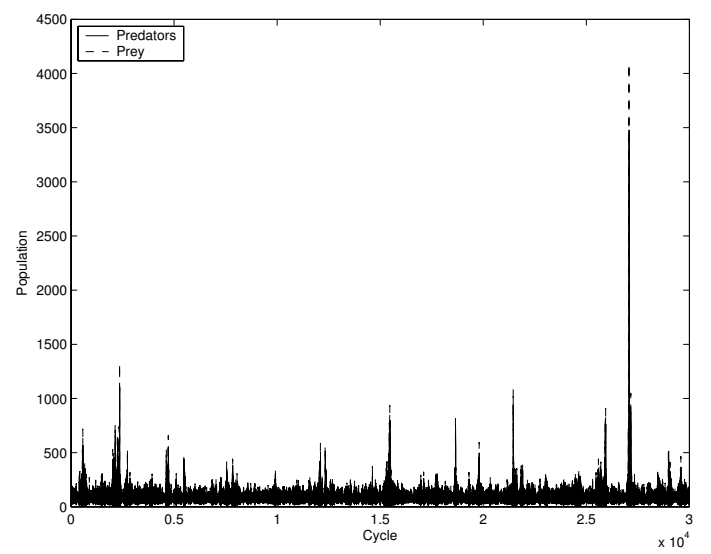

Figure 2: The individual-based model-30 000 cycles.

To test the effects of between-generational timing in the model, the algorithm was modified so that the prey population variable is held constant for the purpose of calculating the probability of eating until the end of each cycle, meaning that within each cycle each predator has the same probability of eating rather than diminishing chances as prey are consumed. The output from the model thus modified is displayed in Figure 3, which was started with the same parameter set used to generate Figures 1 and 2 and is typical of the behavior of the model with this change. The most significant change in the model behavior is that stable cycling populations do not appear: one of the populations always becomes extinct within 100 cycles in the parameter space explored. In addition, the amplitude of the population peaks and troughs for the two species are much closer than when within-generational updating is used, but the period of the population oscillations does not change.

\section{The importance of individual variation}

As described in Chivers and Herbert [2003], the model described here exhibits sensitivity to the level of variation in the simulated populations. The only source of individual variation, apart from age, is the resource level carried by each individual. If the simulation is started without sufficient variation in the resource levels of the individuals, one of the populations quickly becomes extinct. A sufficient level of variation results in a simulation which executes indefinitely and maintains the required level of variation. This dependence on initial variance is consistently observed over the parameter space investigated.

It is difficult to make conclusions about the importance of this finding as the model described is of two generalized species and the parameter values are therefore not derived from empirical data. The importance of variation in 


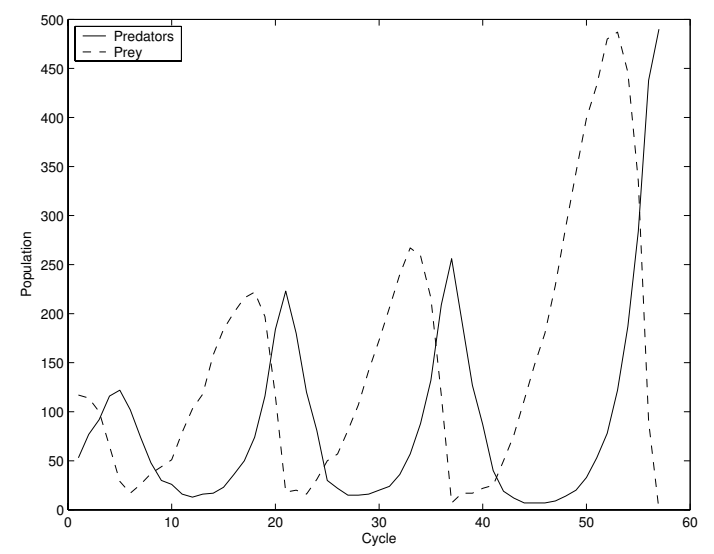

Figure 3: "Between-generational" updating of the prey population variable.

individual-based modeling is emphasized by many researchers, including Connor and White [1999], Uchmański [1999], Aoki and Mizushima [2001], Jager [2001], Cardinale et al. [2002] and Fox and Kendall [2002]. Most of the literature regarding the importance of diversity, however, refers to the number of species, rather than variation within a species [Gaston and Spicer, 1998]. Of the literature which does examine within-species diversity, there is very little research into the importance of physiological or phenotypic diversity due to experience among clonal organisms - the type of diversity in the individual-based model described here and in the model of Grimm and Uchmański [1994].

We add the importance of variation in this IBM to the growing evidence in the literature on individual-based modeling in ecology that individual variation is important to the long-term viability of species in these models. This finding is specifically mentioned by Huston et al. [1988], Schmitz and Booth [1997], Łomnicki [1999] and DeAngelis et al. [2001]. The possible importance of individual-based modeling to ecology because of the possibility that individual variation may be built into the model is of central importance to the work of researchers including Huston et al. [1988], Clark and Rose [1997], Schmitz and Booth [1997], Jager et al. [2000] and DeAngelis et al. [2001].

\section{Discussion}

The findings presented here, of the importance of the timing of system variable updating and the importance of individual variation, support the findings of Grimm and Uchmański [1994] who used an individual based model with significant differences to the model described here. The most significant difference for this discussion is in the assumptions made regarding resource dynamics - the resource inflow and distribution among individuals. 
Grimm and Uchmański [1994] describe an individual-based model of a single population growing over a number of time steps. At pre-determined times the individuals produce a number of offspring depending on the weight of the individual. The individuals grow in an homogeneous environment which has a constant resource inflow. Individuals use resources according to weight and the level of resources available - a deterministic formula. At optimum resource conditions all individuals assimilate resources at a similar rate, but given deteriorating resource conditions the assimilation rates vary according to initial weights, which are distributed symmetrically at the start of the simulation. Deteriorating resource conditions occur as the individuals grow and use more resources, the inflow of which is kept constant. One consequence of this resource flow and usage model is that individuals maintain their initial rankings according to a weight throughout the simulation.

Grimm and Uchmański [1994] present results which demonstrate the importance of the timing of variable updating and of individual variability. As individuals grow towards the time at which they may reproduce, all individuals are immediately affected by the consumption of all other individuals. This within-generational effect is of crucial importance to the model dynamics. Similarly, individual variation in the model results in different life histories - stronger individuals tend to survive and reproduce, while weaker individuals do not reproduce and may die. This variation is important to the density dependence and population self-regulation exhibited by the model.

In contrast to this resource flow system, in the predator-prey model described here resources are allocated to prey individuals randomly, within upper and lower bounds, each cycle. Predators gain resources by eating prey selected at random. This simplified representation of a real system does have some features which are more realistic than the model of Grimm and Uchmański. Inflow is not constant, individual uptake of resources is stochastic and so individual resource levels and growth rates do not maintain their initial rankings.

In both models individuals compete for resources in an homogeneous environment space. Both models favor strong individuals for reproduction, and the model of Grimm and Uchmański also favors strong individuals for feeding.

Despite their fundamental differences, both models successfully produce a mechanism of density-dependent population regulation, resulting in populations which persist over many generations and display the similar characteristics which are the focus of this paper.

\section{Concluding remarks}

We describe here a generalized individual-based model of predator-prey interaction which demonstrates sensitivity to the timing of system-level variable updating and individual variability for the parameter sets used. The significance of our findings is emphasized by the similar findings of Grimm and Uchmański who made different assumptions regarding resource flow. These findings are not commonly discussed in the literature: the importance of variability to the dy- 
namics of individual-based models is occasionally mentioned (examples listed in Section 4), but the significance of within-generational time scales is very rarely addressed.

As in ecological systems, individual interactions in an IBM occur in real-time and have instant effects; the effects are not usually accumulated to be applied at arbitrary time intervals as is the case in top-down models. Grimm and Uchmański [1994] suggest that these time lags in effect in mathematical models may cause the chaotic fluctuations in population observed in some models. Certainly the difference between the between-generational and within-generational effects are important in these two different individual-based models.

The importance of variation is also an important factor in real systems. Interactions between heterogeneous individuals, from which system level outcomes emerge, are of profound importance to dynamics of the models described in this paper. The averaging of individuals in classical modeling may remove some of the important mechanisms of density dependence evident in the individual-based models [Grimm and Uchmański, 1994]. The loss of variance in the populations of these models clearly affects on the dynamics of the models.

\section{Acknowledgments}

The authors gratefully acknowledge the insightful comments of Volker Grimm, which led to the development of this paper.

\section{References}

I. Aoki and T. Mizushima. Biomass diversity and stability of food webs in aquatic ecosystems. Ecological Research, 16:65-71, 2001.

B. J. Cardinale, M. A. Palmer, and S. L. Collins. Species diversity enhances ecosystem functioning through interspecific facilitation. Nature, 415:426-429, 2002 .

W. J. Chivers and R. D. Herbert. The effects of varying parameter values and heterogeneity in an individual-based model of predator-prey interaction. Advances in Complex Systems, 6(3):441-456, 2003.

M. E. Clark and K. A. Rose. Individual-based model of stream-resident rainbow trout and brook char: model description, corroboration, and effects of sympatry and spawning season duration. Ecological Modelling, 94:157-175, 1997.

M. M. Connor and G. C. White. Effects of individual heterogeneity in estimating the persistence of small populations. Natural Resource Modeling, 12(1):109$127,1999$. 
D. L. DeAngelis, W. M. Mooij, M. P. Nott, and R. E. Bennetts. Individualbased models: Tracking variability among individuals. In A. Franklin and T. Shenk, editors, Modeling in Natural Resource Management: Development, interpretation and application, chapter 11, pages 171-195. Island Press, 2001.

G. A. Fox and B. E. Kendall. Demographic stochasticity and the variance reduction effect. Ecology, 83(7):1928-1934, 2002.

K. J. Gaston and J. I. Spicer. Biodiversity: An Introduction. Blackwell Science, 1998.

V. Grimm and J. Uchmański. Ecological systems are not dynamic systems: some consequences of individual variability. In J. Grasman and G. van Straten, editors, Predictability and Nonlinear Modelling in Natural Sciences and Economics, pages 248-259. Kluwer Academic Publishers, Dordrecht, The Netherlands, 1994.

M. Huston, D. L. DeAngelis, and W. Post. New computer models unify ecological theory. BioScience, 38(10):682-691, 1988.

H. I. Jager. Individual variation in life history characteristics can influence extinction risk. Ecological Modelling, 144:61-76, 2001.

H. I. Jager, K. Lepla, J. Chandler, P. Bates, and W. Van Winkle. Population viability analysis of white sturgeon and other riverine fishes. Environmental Science \& Policy, 3:S483-S489, 2000.

A. Łomnicki. Individual-based models and the individual-based approach to population ecology. Ecological Modelling, 115(2-3):191-198, 1999.

O. J. Schmitz and G. Booth. Modelling food web complexity: The consequences of individual-based, spatially explicit behavioural ecology on trophic interactions. Evolutionary Ecology, 11(4):379-398, 1997.

J. Uchmański. What promotes persistence of a single population: an individualbased model. Ecological Modelling, 115:227-241, 1999. 